\title{
Synthesis of polyaniline nanotubes through UV light catalytic method
}

\author{
SUN CHUANYU*, WANG YU \\ Department of Materials Chemistry, Yinuo Institution, Changchun, 130033, China
}

\begin{abstract}
In this study, nitrocellulose (NC) fiber blanket prepared by electrostatic spinning method has been used as a template, and copper nitrate $\left(\mathrm{Cu}\left(\mathrm{NO}_{3}\right)_{2}\right)$ as an oxidant to synthesise polyaniline nanotubes doped with heteropolyacid $\left(\mathrm{H}_{4} \mathrm{SiW}_{12} \mathrm{O}_{40}, \mathrm{SiW}_{12}\right)$ using UV light catalytic method. Infrared spectroscopy (IR), X-ray powder diffraction (XRD), scanning electron microscopy (SEM) and transmission electron microscopy (TEM) technologies were applied to characterize the prepared samples of polyaniline nanotubes. The results show that the external diameter of the tube is about $200 \mathrm{~nm}$, and the internal diameter about $170 \mathrm{~nm}$. We also give a reasonable speculation and explanation about the formation mechanism of the nanotubes.
\end{abstract}

Keywords: UV irradiation; polyaniline; nanotubes; template; heteropolyacid

(C) Wroclaw University of Technology.

\section{Introduction}

Conducting polyaniline (PANI) has been widely investigated for thirty years due to its easy preparation, good environmental stability, low cost and unique doping mechanism. It has already become one of the focus in the conductive polymers family. In recent years, one-dimensional polyaniline nanotubes, as a new important branch of polyaniline materials, have caused great concern among scientists around the world and become a new hotspot of research. For example, Martin from United States, Greiner from Germany [1] and Wan Meixiang from China [2] have done a lot of studies on polyaniline nanotubes. Our group also used the template method to prepare polyaniline nanotube materials in the past years [3-6].

Electrospinning is a simple and common technology that has been widely studied for the use of spinning of a polymer solution. In a typical spinning process, the liquid polymer under electric field is extruded from a nozzle of a syringe, forming tiny droplets. When the electric field reaches a certain intensity, the charges gather on the surface of the droplets which allows them to overcome the

\footnotetext{
*E-mail: chuanyuvip@tom.com
}

surface tension of the liquid, resulting in the formation of ejection stream from small droplets. The ejection stream is accelerated and sprayed towards a receiver. With the evaporation of the solvent, the jet length is elongated and becomes several times longer than its original length [7]. In this way continuous ultra-fine polymer fibers can be obtained. $\mathrm{NC}$ is a white and water-insoluble solid, and it has excellent solubility in a solution of acetone and ethylene. While in an acetone solution it also has very good adhesion ability, the mixed solution can be directly used for electrospinning. By changing the factors, such as applied voltage, the solution concentration, the distance between the receiving plate and injection port, and mixing time we can change the thickness of the fibers. The diameter of electrospinned fibers generally varies between microscale to nanoscale dimensions.

Based on previous studies [8,9], $\mathrm{Cu}\left(\mathrm{NO}_{3}\right)_{2}$ was used as an oxidant, $\mathrm{SiW}_{12}$ as a dopant and $\mathrm{NC}$ as a template. Polyaniline nanotubes doped with $\mathrm{SiW}_{12}$ were successfully synthesized under ultraviolet irradiation. We studied the relationship between the fiber diameter and the inner diameter of the nanotubes and also the formation mechanism of the nanotubes. 


\section{Experiments}

\subsection{Reagents and instruments}

\section{Reagents}

Aniline (after distillation treatment), $\mathrm{SiW}_{12} \cdot \mathrm{H}_{2} \mathrm{O}, \mathrm{Cu}\left(\mathrm{NO}_{3}\right)_{2}$, acetone and nitrocellulose (NC), used in the experiment were of analytical grade.

\section{Instruments and methods used for characteriza- tion}

- Electrostatic high voltage device and a nozzle (self-assembly);

- UV light source (wavelength of $254 \mathrm{~nm}$ );

- FT-IR spectrum: Alpha-Centauri 650 spectrometer with a $\mathrm{KBr}$ pellet (Nicolet);

- Powder X-ray diffraction (PXRD): D/MAX-2200/PC X-ray diffractometer (Rigaku);

- Scanning electron microscopy (SEM): XL30 ESEM FEG (Philips);

- Transmission electron microscopy (TEM): JEM- 2010 (JEOL).

\subsection{Preparation of nitrocellulose fiber blanket}

$1.0 \mathrm{~g}$ of nitrocellulose was dissolved in $5 \mathrm{~mL}$ of acetone. $1 \mathrm{~mL}$ of $\mathrm{N}, \mathrm{N}$-dimethylformamide (DMF) was added dropwise to the solution. The mixed solution was stirred at room temperature until its composition became homogeneous.

In our experiment, high voltage electrostatic DC device was applied at the testing voltage of $12 \mathrm{kV}$. The distance from the nozzle to the receiving plate was $22 \mathrm{~cm}$. In order to ensure the fibers to be easily peeled off from the receiving plate, a $15 \times 15 \mathrm{~cm}$ steel mesh with small holes was used as the receiving plate. Diagram of a system with a high voltage electrostatic device is shown in Fig. 1.

The prepared nitrocellulose solution was placed in a syringe, and the high-voltage DC device was applied for electrostatic spinning. Finally, the obtained fiber blanket was dried under vacuum for $4 \mathrm{~h}$ at temperature about $60{ }^{\circ} \mathrm{C}$.

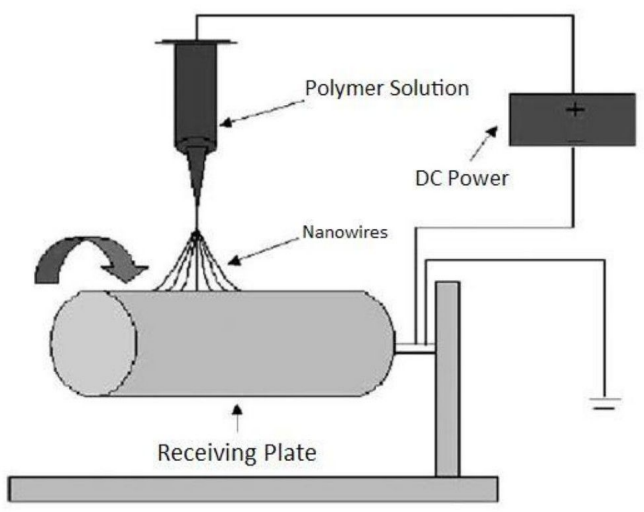

Fig. 1. Diagram of a system with a high voltage electrostatic device.

\subsection{Synthesis of polyaniline nanotubes us- ing nitrocellulose fiber as a template}

$0.5 \mathrm{~g} \mathrm{H}_{4} \mathrm{SiW}_{12} \mathrm{O}_{40}$ was dissolved in $20 \mathrm{~mL}$ of distilled water, the mixed solution was used as a doping acid. $0.1 \mathrm{~g}$ of nitrocellulose fibers were immersed in the solution under ultrasonic agitation for $30 \mathrm{~min}$ in an ice-water bath.

$24.2 \mathrm{~g} \mathrm{Cu}\left(\mathrm{NO}_{3}\right)_{2}$ was added to the acid solution, where the NC fibers were immersed. Under magnetic stirring, $0.08 \mathrm{~mL}$ of aniline as a monomer was slowly added drop by drop.

The whole reaction system was placed in an ice-water bath. Simultaneously, it was exposed to UV irradiation with a wavelength of $254 \mathrm{~nm}$ for $48 \mathrm{~h}$. The fibers were removed after the reaction and washed with distilled water until $\mathrm{pH}$ reached a value of about 6 to 7 . Then the fibers were placed in an acetone solution with ultrasonic washing for $10 \mathrm{~min}$. The resulting precipitate, after centrifuging, was washed with distilled water and ethanol for several times. Finally, the product was dried under vacuum at $50{ }^{\circ} \mathrm{C}$ for $12 \mathrm{~h}$

\section{Results and discussion}

\subsection{Infrared spectroscopy}

The infrared spectrum of polyaniline nanotubes doped with heteropolyacid is shown in Fig. 2. 




Fig. 2. Infrared spectrum of polyaniline nanotubes doped with heteropolyacid.

In the figure, the characteristic absorption peaks of heteropolyacid have appeared in the range of 700 to $1100 \mathrm{~cm}^{-1}$ : $v_{a s}\left(\mathrm{M}-\mathrm{O}_{d}\right): 967.15 \mathrm{~cm}^{-1} ; v_{a s}$ $\left(\mathrm{X}-\mathrm{O}_{a}\right): \quad 914.50 \quad \mathrm{~cm}^{-1} ; \quad v_{a s} \quad\left(\mathrm{M}-\mathrm{O}_{b}-\mathrm{M}\right)$ : $878.39 \mathrm{~cm}^{-1} ; \quad v_{a s}\left(\mathrm{M}-\mathrm{O}_{c}-\mathrm{M}\right): 789.64 \mathrm{~cm}^{-1}$, respectively. It indicates that the heteropolyacid has been doped in the polyaniline molecule [10]. The peaks at $1553 \mathrm{~cm}^{-1}$ and $1487 \mathrm{~cm}^{-1}$ represent the absorption peaks of quinonediimine and benzene diimine skeleton vibrations. The peak at $1143 \mathrm{~cm}^{-1}$ shows that the polyaniline is in the doped state [11]. In addition, a peak, which has occurred at $1304 \mathrm{~cm}^{-1}$ is attributed to the stretching vibration of $\mathrm{C}-\mathrm{N}$, related to benzene. This also indicates that the prepared product is indeed polyaniline in the doped state. The characteristic peak of nitrocellulose disappears at 1600 to $1655 \mathrm{~cm}^{-1}$, which shows that the nitrocellulose fibers template has been removed from the product.

\subsection{X-ray powder diffraction}

X-ray powder diffraction pattern of the product is shown in Fig. 3.

An analysis of the XRD pattern shows that the product is in a semi-crystalline state. The polyaniline nanotubes prepared by using nitrocellulose fiber blanket as a template have a distinct broad peak at $2 \theta=7.4^{\circ}$ [4]. This indicates that the formation of the doped acid state led to the shortrange ordering of anions along the polyaniline

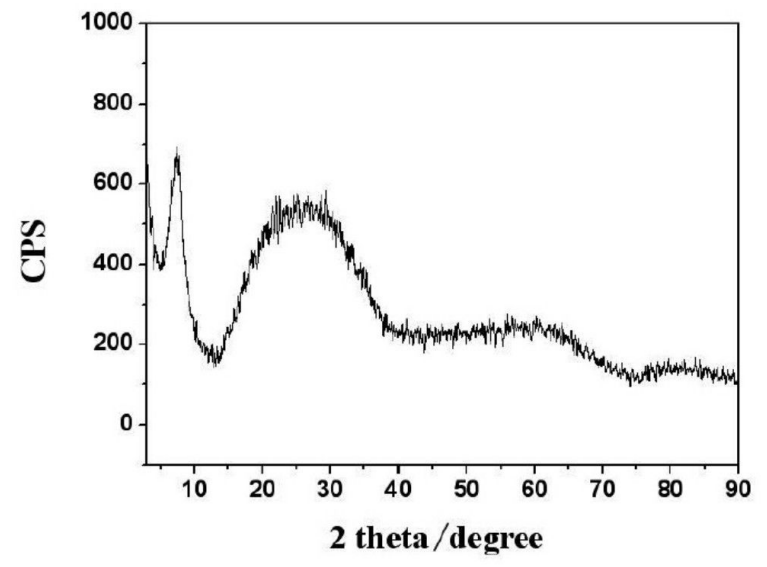

Fig. 3. X-ray powder diffraction pattern of polyaniline nanotubes doped with heteropolyacid.

chain [12]. The broad peak that appeared at $2 \theta=$ $25^{\circ}$ shows that the structure of polyaniline nanotubes is amorphous. The weak broad peak at high angle is the peak, which is vertical to the polyaniline chain [13]. The results of above analysis indicate that the resulting polyaniline fibers are disordered in a long-range, while they are ordered in a short-range.

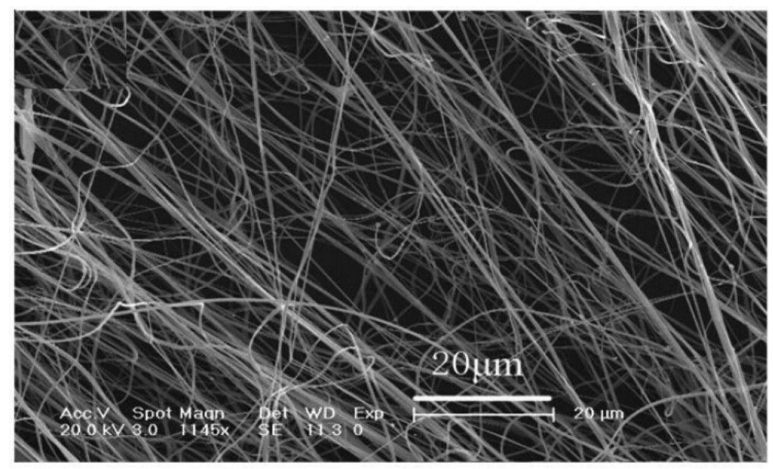

Fig. 4. SEM image of electro-spun NC fibers.

\subsection{Scanning electron micrograph and transmission electron micrograph}

A scanning electron microscope image of the nitrocellulose fibers and a diagram of an average fiber diameter distribution are shown in Fig. 4 and Fig. 5. The average diameter of the fiber is about $170 \mathrm{~nm}$. 




Fig. 5. The distribution diagram of fiber diameter.

Scanning electron micrograph and transmission electron micrograph of the prepared polyaniline nanotubes doped by heteropolyacid, using nitrocellulose fiber blanket as a template are shown in Fig. 6 and Fig. 7.

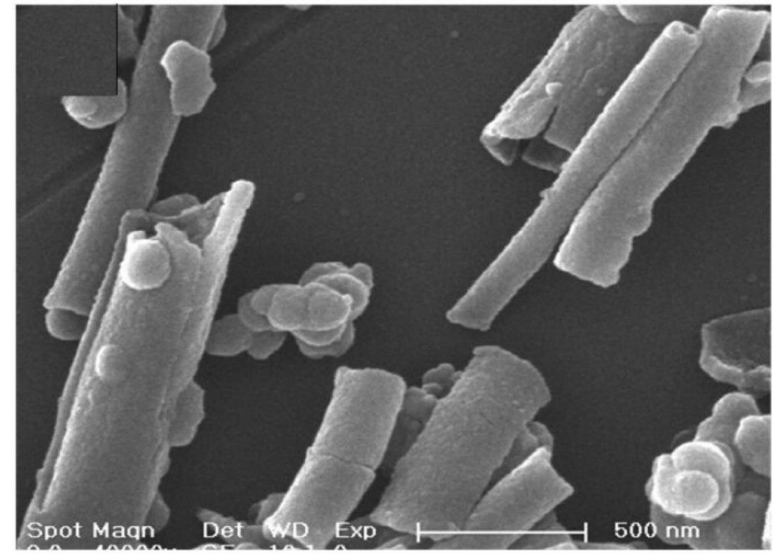

Fig. 6. Scanning electron micrograph of polyaniline nanotubes doped with heteropolyacid.

As can be seen from the figure, the resulting product is essentially regular in shape. The outer diameter of the tube is about $200 \mathrm{~nm}$ and the inner diameter is about $170 \mathrm{~nm}$. Obviously, the inner diameter of the fibers depends on the thickness of NC. This is presumably due to the fact that nitrocellulose fibers have a large surface area, therefore, the heteropolyacid is mainly adsorbed on the wire around the nitrocellulose. Polymerization of aniline needs acidic environment, therefore, aniline mainly



Fig. 7. Transmission electron micrograph of polyaniline nanotubes doped with heteropolyacid.

takes fibers as a template and polymerization takes places on its surface [14].

The presented results show that the method of dissolving and removing the center template can be used to prepare polyaniline nanotubes with an intact morphology. Presumably, this method is also applicable for other soluble templates to prepare tubular polyaniline.

\section{Formation mechanism}

In our experiment, $\mathrm{Cu}\left(\mathrm{NO}_{3}\right)_{2}$ was used as an oxidizing agent and $\mathrm{SiW}_{12}$ as a dopant. The resulting products are not organic-inorganic composite materials, instead they are pure polyaniline. By analyzing the above experiments, we speculate that the formation mechanism is as follows: UV photons interact with the solution, and the aniline monomer is excited to its excited state. Thereby solvated electrons are produced. The solvated electrons can be transferred from aniline under excited state to $\mathrm{Cu}^{2+}$, forming the aniline radical cation and $\mathrm{Cu}^{+}$(because the $\mathrm{Cu}^{2+} / \mathrm{Cu}^{+}$electric potential of the electrode pair is $0.57 \mathrm{~V}$, while $\mathrm{Cu}^{2+} / \mathrm{Cu}$ electric potential of the electrode pair is $0.34 \mathrm{~V}, \mathrm{Cu}^{2+}$ is more easily reduced to $\mathrm{Cu}^{+}$by a weak reducing agent). Polymerization and interactions of aniline radical cation lead to the formation of polyaniline. While $\mathrm{Cu}^{+}$is instable, it can be oxidized by $\mathrm{SiW}_{12}$ to $\mathrm{Cu}^{2+}$ and remains in the 
solution. Therefore, we can obtain pure polyaniline, not composite materials.

\section{Conclusions}

Using NC fiber blanket as a template and $\mathrm{Cu}\left(\mathrm{NO}_{3}\right)_{2}$ as an oxidant, polyaniline nanotube doped with heteropolyacid can be prepared under UV irradiation. We have studied its morphology and characteristics by infrared spectroscopy, $\mathrm{X}$-ray powder diffraction, SEM and TEM characterization. The results show that a specific form of polyaniline can be synthesized by using metal salt as an oxidant and catalysis by UV irradiation.

\section{References}

[1] Dersch R., Steinhart M., Boudriot U., Greiner A., Wendorff J.H., Polym. Adv. Technol., 16 (2005), 276.

[2] Ding H., Shen J., Wan M., Chen Z., Macromol. Chem. Phys., 209 (8) (2008), 864.

[3] Chunnyu S., YU W., Optoelectron. Adv. Mat., 6 (11 12) (2012), 1037.
[4] Chuanyu S., Farabi M.D., Yu W., Optoelectron. Adv. Mat., 8 (7 - 8) (2014), 810.

[5] Chuanyu S., Yu W., Mater. Sci.-Poland, 3 (32) (2014), 419.

[6] Chuanyu S., Yu W., Mater. Sci.-Poland, 4 (32) (2014), 521.

[7] Chen C., Chuanbao C., Xilan M., Yin T., Hesun Z., Polymer, 47 (18) (2006), 6322.

[8] Chuanyu S., Yu W., Optoelectron. Adv. Mat., 6 (7 8) (2012), 771.

[9] Bhattacharya P., Dhibar S., Das C.K., Polymer. Plast. Tech. Eng., 52 (9) (2013), 892.

[10] Ding B., Gong J., Kim J., Shiratori S., Nanotechnology, 16 (6) (2005), 785.

[11] Harada I., Furukawa Y., Ueda F., Synthetic Met., 29 (1) (1989), 303.

[12] Jozefowicz M.E., Epstein A.J., Pouget J.P., Synthetic Met., 41 (1) (1991), 723.

[13] Sun Y., MacDiarmid A.G., Epstein A.J., J. Chem. Soc. Chem. Commun., 7 (1990), 529.

[14] Xue B., QI S., Gong J., J. Nanosci. Nanotechno., 7 (12) (2007), 4515.

Received 2014-10-17

Accepted 2014-11-14 